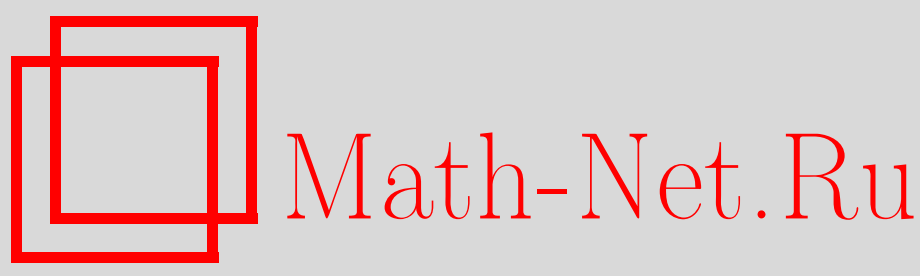

М. И. Зеликин, Связность, порождаемая задачей минимизации кратного интеграла, Матем. сб., 1997, том 188, номер 1, 59-72

DOI: https://doi.org/10.4213/sm187

Использование Общероссийского математического портала Math-Net.Ru подразумевает, что вы прочитали и согласны с пользовательским соглашением

http://www . mathnet.ru/rus/agreement

Параметры загрузки:

IP: 54.162 .127 .20

26 апреля 2023 г., 12:58:46 
УДК 519.9

\author{
М.И. Зеликин
}

\title{
Связность, порождаемая задачей минимизации кратного интеграла
}

Рассматривается функционал, квадратично зависящий от сечений векторного расслоения $\pi: \xi \rightarrow \mathfrak{N}$ над гладким многообразием $\mathfrak{N}$, назьваемьпй интегралом Дирихле. Получена и исследована квадратичная система уравнений в частных производных, которая по аналогии с одномерньм случаем назьвается уравнением Риккати. Показано, что решения этой системы определяют связность $\nabla$ на расслоении $\xi$. Поле экстремалей для функционала Дирихле существует тогда и только тогда, когда найдется решение уравнения Риккати, определяющее плоскую связность. Глобально определенное решение уравнения Риккати, удовлетворяющее некоторьм дополнительным условиям, обеспечивает положительную определенность функционала Дирихле.

Библиограффия: 9 названий.

Задача минимизации кратного интеграла возникла из работ Плато по минимальным поверхностям. Кроме того, к задаче минимизации кратного интеграла приводят вариационные принципы для сплошной среды (теории упругости и пластичности, теория оболочек, квантовая теория поля и т. д.). Рассмотрим кратньй интеграл как функционал, заданный на подмногообразиях риманова пространства, по которым производится интегрирование. Граница подмногообразий для простоты предполагается фиксированной. Вторая вариация этого интеграла есть квадратичный функционал, называемый функционалом Дирихле. Для того чтобы его определить в общем случае, надо рассмотреть нормальное расслоение к варьируемому подмногообразию и в качестве вариаций этого подмногообразия взять сечения нормального расслоения. В результате мы приходим к следуюшей конструкции.

Пусть $\mathfrak{N}$ - гладкое $\nu$-мерное риманово многообразие с краем $\partial \mathfrak{N} ; \pi: \xi \rightarrow \mathfrak{N}-$ векторное расслоение над базой $\mathfrak{N}$. Слой $\pi^{-1} t$, т.е. полный прообраз точки $t \in \mathfrak{N}$ при отображении $\pi$, есть $n$-мерное линейное пространство, обозначаемое через $F_{t}$. Локальные координаты на $\mathfrak{N}$ будут обозначаться через $t=\left(t^{1}, \ldots, t^{\nu}\right)$, координаты на слоях - через $x=\left(x^{1}, \ldots, x^{n}\right)$.

Рассмотрим квадратичный интегральный функционал (функционал Дирихле)

$$
\mathscr{J}=\int_{\mathfrak{N}} F d t
$$

где функция $F$ в локальных координатах имеет вид

$$
F=a_{i j}^{\alpha \beta}(t) \frac{\partial x^{i}}{\partial t^{\alpha}} \frac{\partial x^{j}}{\partial t^{\beta}}+2 c_{i j}^{\alpha}(t) \frac{\partial x^{i}}{\partial t^{\alpha}} x^{j}+b_{i j}(t) x^{i} x^{j} ;
$$

Работа выполнена при финансовой поддержке Российского фонда фундаментальных исследований (грант № 96-01-01360). 
$d t$ есть элемент объема многообразия $\mathfrak{N}: d t=d t^{1} \wedge \cdots \wedge d t^{\nu}, a_{i j}^{\alpha \beta}, c_{i j}^{\alpha}, b_{i j}$ - гладкие функции, заданные на многообразии $\mathfrak{N}$. Здесь и в дальнейшем используется соглашение о суммировании по повторяюшимся индексам, причем латинские индексы относятся к координатам на слоях и пробегают значения от 1 до $n$; греческие индексы относятся к координатам на базе и пробегают значения от 1 до $\nu$. Функционал $\mathscr{J}$ рассматривается на пространстве $C^{\infty}(\xi)$-гладких сечений $x(\cdot)$ расслоения $\xi$, удовлетворяющих граничным условиям

$$
\left.x\right|_{\partial \mathfrak{N}}=0
$$

Допуская некоторую вольность в обозначениях, будем в дальнейшем записывать подынтегральное выражение в локальной форме, как если бы интегрирование проводилось в одной карте, хотя, на самом деле, интегрирование происходит по всему многообразию $\mathfrak{N}$. Восходлщая к работе Клебша [1] идея исследования функционала Дирихле состоит в его сведении (с помощью надлежащего выбора представителя в соответствуюшем классе когомологий) к интегралу от главных членов подынтегрального выражения:

$$
\int_{\mathfrak{N}} a_{i j}^{\alpha \beta}(t)\left(\frac{\partial x^{i}}{\partial t^{\alpha}}-w_{\alpha k}^{i}(t) x^{k}\right)\left(\frac{\partial x^{j}}{\partial t^{\beta}}-w_{\beta k}^{j}(t) x^{k}\right) d t=\int_{\mathfrak{N}} a_{i j}^{\alpha \beta} y_{\alpha}^{i} y_{\beta}^{j} d t
$$

где $y_{\alpha}^{i}=\frac{\partial x^{i}}{\partial t^{\alpha}}-w_{\alpha k}^{i} x^{k}$, а через $w_{\alpha k}^{i}(t)$ обозначены функции, которые мы будем подбирать. А именно, прибавим под знаком интеграла (1) замкнутую дифференциальную форму. Легко видеть, что вследствие стягиваемости слоев векторного расслоения и в силу граничных условий (2) значение интеграла от этого не изменится. Чтобы структура подынтегрального выражения не изменилась, прибавляемое выражение (так же как и исходное подынтегральное выражение в функционале $\mathscr{J}$ ) должно быть квадратичной по переменным $x, \frac{\partial x}{\partial t}$, горизонтальной дифференциальной формой порядка $\nu$ на пространстве $\mathfrak{J}^{1} \xi-1$-джетов расслоения $\xi$. Напомним, что дифференциальная форма на пространстве 1-джетов над $\xi$ называется горизонтальной, если она обращается в нуль на векторах слоя расслоения $\mathfrak{J}^{1} \xi \rightarrow \xi$, иными словами, если она зависит от дифференциалов координат только самого расслоения $\xi$.

Уравнение Эйлера для функционала (1) выглядит следующим образом

$$
-\frac{\partial}{\partial t^{\alpha}}\left(a_{i j}^{\alpha \beta} \frac{\partial x^{j}}{\partial t^{\beta}}+c_{i j}^{\alpha} x^{j}\right)+\left(c_{j i}^{\alpha} \frac{\partial x^{j}}{\partial t^{\alpha}}+b_{i j} x^{j}\right)=0 .
$$

ЛЕмма 1. Уравнение Эйлера для функиионала (1) тождественно выполняется тогда и только тогда, когда подынтегральное выражсение мохсет быть записано в виде замкнутой дифференциальной формы $\theta$ на расслоении $\xi$.

ДокАЗАТЕЛЬство. Покажем сначала, что из тождественного выполнения уравнения Эйлера следует, что подынтегральное выражение (1) может быть записано в виде дифференциальной формы на $\xi$. Обозначим через $\{d t\}_{\alpha}$ внешнее произведение всех дифференциалов $d t^{\gamma}$ за исключением одного сомножителя 
$d t^{\alpha}$, а через $\{d t\}_{\alpha \beta}$ - внешнее произведение всех дифференциалов $d t^{\gamma}$ за исключением двух сомножителей $d t^{\alpha}$ и $d t^{\beta}$ (порядок сомножителей предполагается при этом естественным). Слагаемые подьнтегрального выражения вида $b_{i j} x^{i} x^{j} d t$ и $c_{i j}^{\alpha} \frac{\partial x^{i}}{\partial t^{\alpha}} x^{j} d t=(-1)^{\alpha-1} c_{i j}^{\alpha} x^{j} d x^{i} \wedge\{d t\}_{\alpha}$ уже имеют вид дифференциальных форм на $\xi$. Коэффициенты при главных членах подынтегрального выражения $a_{i j}^{\alpha \beta}$ должны быть кососимметричны по индексам $\alpha, \beta$, так как в противном случае старшие члены уравнения Эйлера $a_{i j}^{\alpha \beta} \frac{\partial^{2} x^{j}}{\partial t^{\alpha} \partial t^{\beta}}$ не обратятся тождественно в нуль. Поскольку эти коэффициенты симметричны относительно перестановок пар индексов $(i, \alpha)$ и $(j, \beta)$, они должны быть кососимметричны и по индексам $i, j$. Следовательно, главный член в функционале (1) может быть записан в виде дифференциальной формына $\xi$

$$
(-1)^{\alpha+\beta} a_{i j}^{\alpha \beta} d x^{i} \wedge d x^{j} \wedge\{d t\}_{\alpha \beta} .
$$

Для того чтобы доказать, что образовавшаяся под знаком интеграла дифференциальная форма $\theta$ замкнута, рассмотрим произвольное сечение $x(\cdot)$ расслоения $\xi$ и его вариацию $h(\cdot)$ такую, что $\left.h\right|_{\partial \mathfrak{N}}=0$. Поскольку расслоение $\xi$ векторное и его слои стягиваемы, многообразия, определяемые сечениями $x(\cdot)$ и $x(\cdot)+h(\cdot)$, гомотопны. Тождественное выполнение уравнения Эйлера означает, что производная функционала (1), вычисленная на любом сечении $x(\cdot)$ расслоения $\xi$ по любому направлению $h(\cdot)$, удовлетворяющему нулевым граничным условиям, равна нулю. Поэтому в процессе гомотопии значение функционала остается постоянным:

$$
\int_{x(\cdot)} \theta=\int_{x(\cdot)+h(\cdot)} \theta .
$$

Многообразия $x(\cdot)$ и $x(\cdot)+h(\cdot)$, удовлетворяя одним и тем же граничньп условиям $(2)$, ограничивают некое $(\nu+1)$-мерное многообразие $\mathfrak{M}$, и в силу формулы Стокса $\int_{\mathfrak{M}} d \theta=0$. Так как сечения $x(\cdot)$ и $x(\cdot)+h(\cdot)$ произвольны, отсюда следует, что форма $\theta$ замкнута.

Обратно, из замкнутости формы $\theta$ следует, что

$$
\int_{x(\cdot)} \theta=\int_{x(\cdot)+h(\cdot)} \theta
$$

и отсюда легко получается, что производная функционала $\mathscr{J}$ на любом сечении $x(\cdot)$ расслоения $\xi$ по любому направлению $h(\cdot)$ равна нулю, что и означает тождественное вьполнение уравнения Эйлера.

В силу леммы 1 для сводимости функционала (1) к виду (3) надо потребовать, чтобы результаты применения оператора Эйлера (т.е. правой части уравнений (4)) к обоим подынтегральным выражениям тождественно совпадали

$$
\begin{aligned}
& -\frac{\partial}{\partial t^{\alpha}}\left(a_{i j}^{\alpha \beta} \frac{\partial x^{j}}{\partial t^{\beta}}+c_{i j}^{\alpha} x^{j}\right)+\left(c_{j i}^{\alpha} \frac{\partial x^{j}}{\partial t^{\alpha}}+b_{i j} x^{j}\right) \\
& \quad \equiv-\frac{\partial}{\partial t^{\alpha}}\left(a_{i j}^{\alpha \beta} \frac{\partial x^{j}}{\partial t^{\beta}}-a_{i j}^{\alpha \beta} w_{\beta k}^{j} x^{k}\right)+\left(-a_{k j}^{\alpha \beta} \frac{\partial x^{j}}{\partial t^{\beta}} w_{\alpha i}^{k}+w_{\alpha i}^{k} a_{k j}^{\alpha \beta} w_{\beta s}^{j} x^{s}\right) .
\end{aligned}
$$


Члены со вторыми производными взаимно уничтожаются. Приравнивая коэффициенты при $\frac{\partial x^{j}}{\partial t^{\alpha}}$, получаем равенства

$$
a_{i l}^{\alpha \beta} w_{\beta j}^{l}+c_{i j}^{\alpha}=a_{k j}^{\beta \alpha} w_{\beta i}^{k}+c_{j i}^{\alpha}
$$

Приравнивая коэффициенты при $x^{j}$, получаем равенства

$$
\frac{\partial}{\partial t^{\alpha}}\left(a_{i k}^{\alpha \beta} w_{\beta j}^{k}+c_{i j}^{\alpha}\right)=b_{i j}-a_{k s}^{\alpha \beta} w_{\alpha i}^{k} w_{\beta j}^{s} .
$$

Резюмируем предыдущие рассуждения в виде теоремы.

ТЕОРема 1. Для того чтобы функиионал Дु допускал сведение к виду (3), необходимо и достаточно, чтобь существовало глобальное (т.е. определенное на всем многообразии $\mathfrak{N})$ решение системы (5), (6).

Для тензоров, индексы которых меняются от 1 до $n$, мы введем матричные обозначения, используя заглавные буквы, соответствующие исходным строчным. Например, при фиксированных $\alpha, \beta$ матрищу с элементами $a_{i j}^{\alpha \beta}$ мы обозначим через $A^{\alpha \beta}$ и т. д. Транспонирование матрицы обозначается индексом $T$. Используя соотношения $a_{k j}^{\alpha \beta}=a_{j k}^{\beta \alpha}$, перепишем уравнения (5) в виде

$$
A^{\alpha \beta} W_{\beta}+C^{\alpha}=\left[A^{\alpha \beta} W_{\beta}+C^{\alpha}\right]^{T},
$$

а уравнения (6) в виде

$$
\frac{\partial}{\partial t^{\alpha}}\left[A^{\alpha \beta} W_{\beta}+C^{\alpha}\right]=B-W_{\alpha}^{T} A^{\alpha \beta} W_{\beta} .
$$

Уравнение (8) с условием симметрии (7) играет для задачи минимизации кратного интеграла ту же роль, что и дифференциальное уравнение Риккати для задачи минимизации однократного интеграла. А именно, справедлива следуюшая

ТЕОРема 2. Предположим, что көадратичная форма $a_{i j}^{\alpha \beta} y_{\alpha}^{i} y_{\beta}^{j}$, заданная на $(n \times \nu)$-матрицах $y_{\alpha}^{i}$, неотрицательно определена при всех $t \in \mathfrak{N}$. Пусть существует глобальное (т.е. определенное на всем многообразии $\mathfrak{N}$ ) решение системы (7), (8). Тогда функиионал (1), (2) неотрицательно определен. Существование решения системы (7), (8), определенного на области $\mathfrak{D} \subset \mathfrak{N}$, влечет за собой локальную неотрицательную определенность на любой подобласти $\mathfrak{O}^{\prime} \subset \mathfrak{O}$, m.е. условие $\int_{\mathfrak{O}^{\prime}} F d t \geqslant 0$.

Доказательство непосредственно следует из теоремы 1 и формулы (3).

ЗАмечАниЕ. Теорема 2 дает нетривиальный результат даже в случае однократного интеграла $(\nu=1)$. Дело в том, что при выводе уравнения (8) не накладывалось никаких ограничений на матрищы $A^{\alpha \beta}$. Следовательно, теорема 2 при $\nu=1$ применима также и в случае, когда матрица $A$ вырождается. Это дает возможность по новому взглянуть на результаты работ, в которых выписывались 
условия локальной оптимальности функционала $\mathscr{J}$ при вырождении условия Лежандра в изолированной точке $t=\tau$. Например, в работе А. В. Арутюнова [2] выписьвались условия локальной неотрицательности функционала Дирихле в терминах асимптотики функций $a_{i j}, b_{i j}, c_{i j}$ при $t \rightarrow \tau$. Можно показать, что полученные в этой работе условия эквивалентны условию сушествования решения уравнения (8), которое было бы ограниченньм в некоторой окрестности особой точки $t=\tau$. Но самое главное, теорема 2 дает возможность исследовать функционал Дирихле без каких-либо дополнительных предположений о характере вырождения условия Лежандра.

Итак, для изучения вопроса о положительной определенности функционала Дирихле надо сначала попытаться исправить главные члены $a_{i j}^{\alpha \beta} \frac{\partial x^{i}}{\partial t^{\alpha}} \frac{\partial x^{j}}{\partial t^{\beta}}$ так, чтобы они удовлетворяли условиям теоремы 2 , с помошью добавления замкнутой дифференциальной формы $r_{i j}^{\alpha \beta}(t) d x^{i} \wedge d x^{j} \wedge\{d t\}_{\alpha \beta}$. Можно, например, взять $r_{i j}^{\alpha \beta}$ постоянньпии.

ПрЕДПОЛОЖЕНИЕ 1. Существуют $r_{i j}^{\alpha \beta}(t)$, соответствующие замкнутой дифференциальной форме $r_{i j}^{\alpha \beta}(t)\{d t\}_{\alpha \beta}$, такие, что квадратичная форма

$$
a_{i j}^{\alpha \beta} y_{\alpha}^{i} y_{\beta}^{j}+r_{i j}^{\alpha \beta}\left(y_{\alpha}^{i} y_{\beta}^{j}-y_{\beta}^{i} y_{\alpha}^{j}\right)
$$

принимает неотрицательные значения на любых матрицах $y_{\alpha}^{i}$.

Это предположение довольно жесткое, так как необходимым условием неотрицательной определенности функционала (1) является условие Адамара: квадратичная форма $\mathfrak{A}=a_{i j}^{\alpha \beta} y_{\alpha}^{i} y_{\beta}^{j}$, заданная на $(n \times \nu)$-матрицах $y_{\alpha}^{i}$, неотрицательна на конусе матрищ ранга 1, или, иньми словами, биквадратичная форма

$$
a_{i j}^{\alpha \beta} \eta^{i} \eta^{j} \lambda_{\alpha} \lambda_{\beta} \geqslant 0
$$

неотрицательна на всех парах векторов $\eta=\left(\eta^{1}, \ldots, \eta^{n}\right), \lambda=\left(\lambda_{1}, \ldots, \lambda_{\nu}\right)$.

Заметим, что предположение 1 выполняется далеко не всегда даже для одной точки. Об этом свидетельствует результат Терпстра [3], который рассматривал следуюшую алгебраическую задачу: моэнн ли превратить квадратичную форму, заданную на линейном пространстве $(n \times \nu)$-матрии и положительно определенную на конусе матрии ранга 1 , в полохительно определенную форму на всем пространстве матрии, добавляя к ней члены $r_{i j}^{\alpha \beta}\left(y_{\alpha}^{i} y_{\beta}^{j}-y_{\beta}^{i} y_{\alpha}^{j}\right)$, где тензоры $r_{i j}^{\alpha \beta}$ кососимметричнье как по индексам $\alpha, \beta$, так и по индек$c a \mathcal{M} i, j$ (т.е. члены, обрашаюшиеся в нуль на матрицах ранга 1)? Терпстра доказал, что ответ положителен в случае, когда либо $n$, либо $\nu$ не превосходит 2 . В противном случае ответ отрицательный: Терпстра привел пример квадратичной формы для $n=\nu=3$, которая не может быть преврашена в положительно определенную с помощью вышеописанной процедуры. Используя конструкцию Терпстра, Матюхин [4] доказал, что при $\min (n, \mu) \geqslant 3$ множество таких форм открыто.

При вьполнении предположения 1 можно искать глобально определенное решение уравнений $(7),(8)$, и если таковое найдется, то мы окажемся в сфере применимости теоремы 2. В противном случае нам придется рассматривать задачу с 
учетом дифференциальных связей на матрицы $y_{\alpha}^{i}$, которые будут получены ниже в теореме 6.

Как и в случае однократного интеграла [5], уравнение (8) тесно связано с уравнением Эйлера (4), которое в матричной форме имеет вид

$$
-\frac{\partial}{\partial t^{\alpha}}\left[A^{\alpha \beta} \frac{\partial U}{\partial t^{\beta}}+C^{\alpha} U\right]+\left[\left(C^{\alpha}\right)^{T} \frac{\partial U}{\partial t^{\alpha}}+B U\right]=0
$$

где $U-(n \times \nu)$-матрица, соответствующая $n$-параметрическому семейству экстремалей или, иньми словами, полю экстремалей для функционала (1).

ОПРЕДЕЛЕниЕ 1. Решение уравнения (10) называется невырожсденным в $\mathfrak{N}$, если $\operatorname{det} U(t) \neq 0$ при всех $t \in \mathfrak{N}$.

ОПРЕДЕЛЕНИЕ 2. Будем говорить, что решение $U(t)$ уравнения $(10)$ удовлетворяет условию симметрии, если матрица

$$
A^{\alpha \beta} \frac{\partial U}{\partial t^{\beta}} U^{-1}+C^{\alpha}
$$

симметрическая.

ЗАМЕчАНИЕ. В случае однократного интеграла условие симметрии - это условие лагранжевости, т.е. условие симметричности матрицы, задающей координаты $n$-мерного линейного подпространства.

ТЕОРема 3. Предположсим, что $U(t)$ - решение уравнения (10), удовлетворяющее в $\mathfrak{N}$ условиям невырожсденности и симметрии. Тогда

$$
W_{\alpha}=\frac{\partial U}{\partial t^{\alpha}} U^{-1}
$$

удовлетворяет уравнениям (7), (8).

Доказательство сводится к прямой проверке. Условие симметрии очевидно совпадает с условием (7). Проверяем выполнение уравнения (8):

$$
\begin{aligned}
\frac{\partial}{\partial t^{\alpha}} & {\left[A^{\alpha \beta} \frac{\partial U}{\partial t^{\beta}} U^{-1}+C^{\alpha}\right] } \\
& =\frac{\partial}{\partial t^{\alpha}}\left\{\left[A^{\alpha \beta} \frac{\partial U}{\partial t^{\beta}}+C^{\alpha} U\right] U^{-1}\right\} \\
& =\left[\left(C^{\alpha}\right)^{T} \frac{\partial U}{\partial t^{\alpha}}+B U\right] U^{-1}-\left[A^{\alpha \beta} \frac{\partial U}{\partial t^{\beta}}+C^{\alpha} U\right] U^{-1} \frac{\partial U}{\partial t^{\alpha}} U^{-1} \\
& =\left(C^{\alpha}\right)^{T} W^{\alpha}+B-\left[A^{\alpha \beta} W_{\beta}+C^{\alpha}\right] W_{\alpha} .
\end{aligned}
$$

Используя условие (7), получаем

$$
\frac{\partial}{\partial t^{\alpha}}\left[A^{\alpha \beta} \frac{\partial U}{\partial t^{\beta}} U^{-1}+C^{\alpha}\right]=B-W_{\beta}^{T} A^{\alpha \beta} W_{\alpha},
$$

что и дает уравнение (8). 
В отличие от ситуации, соответствующей однократному интегралу, обратное не верно. Не всякое решение уравнения (7), (8) дает решение уравнения Эйлера (9). Для этого необходимо дополнительное условие, которое мы будем называть условием интегрируемости. Рассмотрим соотношения (11) как систему уравнений относительно матрицы $U(t)$ при заданных $W_{\alpha}(t)$ :

$$
\frac{\partial U}{\partial t^{\alpha}}=W_{\alpha} U
$$

Задача Коши для уравнения (12) заключается в том, чтобы найти его решение, удовлетворяюшее условию $U\left(t_{0}\right)=U_{0}$.

Лемма 2. Пусть $\operatorname{det} U_{0} \neq 0$. Тогда задача Коши для уравнений (12) локально разрешима тогда и только тогда, когда

$$
\frac{\partial}{\partial t^{\alpha}} W_{\beta}-\frac{\partial}{\partial t^{\beta}} W_{\alpha}-\left[W_{\alpha}, W_{\beta}\right]=0 .
$$

Здесь $\left[W_{\alpha}, W_{\beta}\right]=W_{\alpha} W_{\beta}-W_{\beta} W_{\alpha}-$ коммутатор матриц $W_{\alpha}$ и $W_{\beta}$.

ДокАзАТЕЛьство. Локальная разрешимость системы (12) эквивалентна замкнутости матричной дифференциальной формы

$$
d U-W_{\alpha}(t) U d t^{\alpha}
$$

Коэффициент при $d t^{\alpha} \wedge d t^{\beta}$ у внешнего дифференциала этой формы равен

$$
\left(\frac{\partial W^{\alpha}}{\partial t^{\beta}}-\frac{\partial W^{\beta}}{\partial t^{\alpha}}-\left[W_{\alpha}, W_{\beta}\right]\right) U=0
$$

Поскольку $\operatorname{det} U(t) \neq 0$ в достаточно малой окрестности точки $t_{0}$, последнее условие эквивалентно (13).

ПРЕДЛОЖЕНИЕ 1. Пусть $W_{\alpha}(\alpha=1, \ldots, \nu)$ непрерывны в $\mathfrak{N}$ и удовлетворяют условию интегрируемости (13). Тогда решение $U(t)$ системы (12) с начальным условием $U\left(t_{0}\right)=U_{0}\left(\operatorname{det} U_{0} \neq 0\right)$ существует, единственно и продолэиимо (как многозначная функиия) на все многообразие $\mathfrak{N}$. При әтом $\operatorname{det} U(t) \neq 0$ при всех $t \in \mathfrak{N}$.

ДокАЗАТЕЛЬСТво. Рассмотрим в $\mathfrak{N}$ произвольную кривую $\gamma(s)$, проходящую через точку $t_{0}$. Система (12) индуцирует линейную систему обыкновенных дифференциальных уравнений от независимого переменного $s$ на кривой $\gamma$. Коэффициенты этой системы непрерьвны, и поэтому ограничение $U(s)=\left.U(t)\right|_{\gamma}$ определено и продолжимо по $s$, причем $\operatorname{det} U(s) \neq 0$. Замкнутость дифференциальной формы (14) гарантирует однозначность $U(t)$ при гомотопии пути $\gamma$, соединяющего точки $t_{0}$ и $t$. При неодносвязном $\mathfrak{N}$ функция $U(t)$ может оказаться многозначной: значение $U(t)$ зависит от гомотопического класса пути $\gamma$.

Для пояснения геометрического смысла уравнения (8) рассмотрим следующую вспомогательную конструкцию. Введем на многообразии Грассмана $G_{n}\left(\mathbb{R}^{n(\nu+1)}\right)$ 
$n$-мерных подпространств пространства $\mathbb{R}^{n(\nu+1)}$ специальную систему координат, являюшуюся векторным аналогом проективных координат на $\mathbb{R P}^{k}$. Рассмотрим в $\mathbb{R}^{n(\nu+1)}$ набор из $(\nu+1) n$-плоскостей, находящихся в общем положении:

$$
T_{0} \oplus T_{1} \oplus \cdots \oplus T_{\nu}=\mathbb{R}^{n(\nu+1)} .
$$

Обозначим через $\pi_{\alpha}$ оператор проектирования на подпространство $T_{\alpha}$ параллельно подпространству $\bigoplus_{\beta \neq \alpha} T_{\beta}$. Пусть $W$ произвольная $n$-мерная плоскость. Если зафиксировать базисы в плоскостях $W$ и $T_{\alpha}(\alpha=0, \ldots, \nu)$, то набор, состоящий из $(\nu+1)(n \times n)$-матриц отображений $\pi_{\alpha}: W \rightarrow T_{\alpha}$ (которые мы также будем обозначать через $\pi_{\alpha}$ ), мы назовем матричными однородными координатами плоскости $W$.

ЗАмЕчАнИЕ. Изменение базиса в плоскости $W$ приводит к умножению всех матриц $\pi_{\alpha}$ справа на одну и ту же невырожденную матрицу.

Рассмотрим карты $M_{\alpha}$ в $G_{n}\left(\mathbb{R}^{n(\nu+1)}\right)$, состоящие из плоскостей $W$, трансверсальных к $\bigoplus_{\beta \neq \alpha} T_{\beta}$. Тогда ограничение проектора $\pi_{\alpha}$ на $W \in M_{\alpha}$ является взаимно однозначным отображением. Матричньми аффинными координатами плоскости $W$ в данной карте будут матрицы

$$
W^{\varkappa}=\pi_{\varkappa} \pi_{\alpha}^{-1}, \quad \varkappa \neq \alpha .
$$

Переход из одной карты в другую описьвается линейным преобразованием пространства $\mathbb{R}^{n(\nu+1)}$, которое в блочной форме (греческие индексы нумеруют блоки, являющиеся $(n \times n)$-матрицами) имеет вид

$$
Y^{\sigma}=\mathfrak{A}_{\varkappa}^{\sigma} X^{\varkappa}
$$

при этом матричные афффинные координаты преобразуются как вектор из обобшенных дробно-линейных преобразований вида

$$
\left(\mathfrak{A}_{1}^{\gamma}+\mathfrak{A}_{\alpha}^{\gamma} W^{\alpha}\right)\left(\mathfrak{A}_{1}^{1}+\mathfrak{A}_{\alpha}^{1} W^{\alpha}\right)^{-1}, \quad \gamma=1, \ldots, \nu
$$

Последняя формула определяет действие $S L(n(\nu+1))$ на $G_{n}\left(\mathbb{R}^{n(\nu+1)}\right)$. Рассматривая это действие как замену переменных в уравнении (8), можно доопределить это уравнение на "бесконечно удаленных" точках карты $M$. Это позволяет рассматривать (8) как уравнение на расслоении $\zeta$, ассоциированном с расслоением 1-джетов $\mathfrak{J}(\xi)$, стандартным слоем которого является многообразие Грассмана $G_{n}\left(\mathbb{R}^{n(\nu+1)}\right)$. Тем самым мы получаем возможность продолжать решения уравнения (8), уходящие на бесконечность, с помощью перехода от карты $M_{0}$ к другой карте на $G_{n}\left(\mathbb{R}^{n(\nu+1)}\right)$.

Решение $W_{\alpha}(t), \alpha=1, \ldots, \nu$, мы будем интерпретировать как связность на расслоении $\xi$.

Напомним, что аффинной связностью $\nabla$ на векторном расслоении $\pi: \xi \rightarrow \mathfrak{N}^{\nu}$ называется гладкое распределение $\nu$-плоскостей (называемых горизонтальными) в касательных пространствах $T_{u} \xi$ к расслоению в точках $u$. Это распределение должно обладать следующим свойством: оператор проектирования касательных векторов к расслоению в точке $u \in \xi$ на слой $F_{\pi u}$ параллельно горизонтальной плоскости линейно зависит от точки $u$ слоя $F_{\pi u}$ (см. [6]-[9]). 
Для упрошения формул мы будем использовать для обозначения касательных векторов не дифференциальные операторы, а двойственные к ним дифференциальные формы, оговаривая каждый раз, что именно имеется в виду: дифференциальная форма (функционал на множестве касательных векторов) или касательньй вектор (двойственньй к ней).

Связность $\nabla$ однозначно определяется дифференциальной формой, определяющей проекцию касательных векторов $d t, d x$ в точке $(t, x)$ на слой. Эта форма должна линейно зависеть от $x$ и давать тождественное отображение на вертикальных векторах $(0, d x)$. Следовательно, она должна иметь вид

$$
\omega=d x-W_{\alpha}(t) x d t^{\alpha} .
$$

Соответствуюший оператор ковариантного дифференцирования по направлению касательного вектора $v$ к многообразию $\mathfrak{N}$ есть

$$
\nabla_{v} x(t)=\frac{\partial x}{\partial t^{\alpha}} v^{\alpha}-W_{\alpha}(t) x v^{\alpha}
$$

Оператор проектирования касательных векторов $(d t, d x)$ в точке $(t, x)$ на слой имеет вид

$$
(d t, d x) \mapsto\left(0, d x-W_{\alpha} x d t^{\alpha}\right) .
$$

Горизонтальньми векторами связности $\nabla$ являются те векторы $(d t, d x)$, которые лежат в ядре этого оператора: $d x-W_{\alpha} x d t^{\alpha}=0$, следовательно, горизонтальная компонента вектора $(d t, d x)$ есть вектор $\left(d t, W_{\alpha} d t^{\alpha}\right)$.

Связность называется плоской, если ее тензор кривизны равен нулю.

ПРЕДЛОЖЕНИЕ 2. Условие интегрируемости (13) выполнено тогда и только тогда, когда связность $\nabla$ плоская.

ДокАЗАТЕЛЬСТво. Вычислим дифференциальную форму кривизны $\Omega$ связности $\nabla$. Имеем

$$
d \omega=-w_{\alpha k}^{i} d x^{k} \wedge d t^{\alpha}-\left(\frac{\partial}{\partial t^{\beta}} w_{\alpha k}^{i}\right) x^{k} d t^{\beta} \wedge d t^{\alpha} .
$$

По определению [6] внешняя ковариантная производная $D \omega$ формы $\omega$ есть значение $d \omega$ на горизонтальных компонентах вектора $(d t, d x)$. Значит

$$
\Omega=D \omega=-w_{\alpha k}^{i}\left(w_{\beta j}^{k} x^{j} d t^{\beta}\right) \wedge d t^{\alpha}-\frac{\partial}{\partial t^{\beta}} w_{\alpha k}^{i} x^{k} d t^{\beta} \wedge d t^{\alpha} .
$$

Коэффициент внешней формы $D \omega$ при $x^{k} d t^{\alpha} \wedge d t^{\beta}$ совпадает с левой частью (14), что и требовалось доказать.

Используя лемму 2 и предложение 2 , можно получить следующий результат.

ТЕОРема 4 (о параметризации). Аффинная связность $\nabla$, определяемая дифференииальной формой $\omega$ является плоской тогда и только тогда, когда существует матричнозначная функиия $U(t)$ такая, что $\operatorname{det} U(t) \neq 0$; $W_{\alpha}(t)=\frac{\partial U}{\partial t^{\alpha}} U^{-1}$. При этом функиия $U(t)$ определена с точностью до умножения справа на постоянную матрицу. 
ДокаЗАтЕльство. Пусть $W_{\alpha}=\frac{\partial U}{\partial t^{\alpha}} U^{-1}$. Тогда в силу леммы 2 вьполнено условие интегрируемости (14), и из предложения 2 следует, что связность $\nabla$ плоская.

Пусть теперь связность $\nabla$ плоская. Тогда система уравнений $\frac{\partial U}{\partial t^{\alpha}}=W_{\alpha} U$ удовлетворяет условиям интегрируемости, и в силу предложения 1 для любого $U_{0}$, $\operatorname{det} U_{0} \neq 0$, сушествует единственное решение $U(t)$ такое, что $U\left(t_{0}\right)=U_{0}$, причем $\operatorname{det} U(t) \neq 0$. Следовательно, $W_{\alpha}=\frac{\partial U}{\partial t^{\alpha}} U^{-1}$ и матрица $U_{0}$ определяет произвол в выборе параметризации $U(t)$.

СЛЕДСТВИЕ. Связность $\nabla$, построенная с помощью решения уравнений (7), (8), порождена невырожденным решением матричного уравнения Эйлера (9) тогда и только тогда, когда она плоская.

Следует добавить, что условие (14) есть в то же время условие интегрируемости связности $\nabla$ или, что то же, условие инволютивности ее горизонтальных плоскостей. А именно, имеет место следующий факт.

УТВЕРЖДЕНИЕ 1. Аффинная связность $\nabla$, определяемая формой $\omega$, интегрируема тогда и только тогда, когда она плоская.

ДокАЗАТЕльство. По теореме Фробениуса векторная дифференциальная форма $\omega$ вполне интегрируема тогда и только тогда, когда ее внешняя производная $\mathrm{d} \omega$ лежит в идеале $I$, порожденном компонентами формы $\omega$. Имея это в виду, запишем $\mathrm{d} \omega$ в виде

$$
\begin{aligned}
\mathrm{d} \omega & =-w_{\alpha k}^{i}\left(d x^{k}-w_{\beta s}^{k} x^{s} d t^{\beta}\right) \wedge d t^{\alpha}-\frac{\partial}{\partial t^{\beta}} w_{\alpha k}^{i} x^{k} d t^{\beta} \wedge d t^{\alpha}-w_{\alpha k}^{i} w_{\beta s}^{k} x^{s} d t^{\beta} \wedge d t^{\alpha} \\
& =-w_{\alpha k}^{i}\left(\omega^{k} \wedge d t^{\alpha}\right)-\left[\frac{\partial}{\partial t^{\alpha}} w_{\beta k}^{i}-\frac{\partial}{\partial t^{\beta}} w_{\alpha k}^{i}+w_{\alpha j}^{i} w_{\beta k}^{j}-w_{\beta j}^{i} w_{\alpha k}^{j}\right] x^{k} d t^{\alpha} \wedge d t^{\beta} .
\end{aligned}
$$

Первое слагаемое в полученном выражении для $\mathrm{d} \omega$ лежит в идеале $I$. Поэтому для того, чтобы форма $\mathrm{d} \omega$ лежала в идеале $I$ (и, тем самым, для интегрируемости связности $\nabla)$, необходимо и достаточно, чтобы $\Omega=0$.

Условие (14) есть следствие сушествования $n$ независимых решений системы уравнений Эйлера (4), которые мы рассматриваем как горизонтальные сечения расслоения $\xi[7]$.

Условие (14) связано также с понятием поля экстремалей. Поле экстремалей это $n$-параметрическое семейство решений уравнений Эйлера такое, что пространство параметров диффеоморфно отображается на слои расслоения $\xi$, на котором определен некоторый инвариантньй интеграл.

Прямым следствием предложений 1 и 2 является следующая

Теорема 5. Решение уравнений (7), (8) порождает поле экстремалей только тогда, когда определяемая этим решением аффинная связность плоская.

Теоремы 2 и 5 показывают, что подход к вопросу о знакоопределенности функционала (1), использующий уравнения (7), (8), предпочтительнее подхода, используюшего поле экстремалей: теорема 2 дает достаточное условие знакоопределенности и при отсутствии поля экстремалей. 
Теорема 1 определяет условия сводимости функционала (1) к виду

$$
\int_{\mathfrak{N}} a_{i j}^{\alpha \beta} y_{\alpha}^{i} y_{\beta}^{j} d t,
$$

где через $y_{\alpha}^{i}$ обозначено

$$
y_{\alpha}^{i}=\frac{\partial x^{i}}{\partial t^{\alpha}}-w_{\alpha k}^{i} x^{k} .
$$

Для того чтобы иметь возможность исследовать функционал (1) в общем случае (т.е. только в предположении (9)), необходимо выяснить, какие функции $y_{\alpha}^{i}$ можно подставлять под знак интеграла (16), если известно, что они получены с помощью соотношений (17). Следовательно, необходимо найти образ дифференциального оператора, стоящего в правой части соотношения (17). Ответ на этот вопрос удобно формулировать в терминах аффинной связности $\nabla$. А именно: в силу (15) правая часть соотношений (17) есть значение ковариантной производной от сечения $x(\cdot)$ на векторе $\partial / \partial t^{\alpha}$.

ОПРЕДЕЛЕНИЕ. Назовем тензорное поле $y_{\alpha}^{i}$ nотенциальным, если оно локально может быть записано как ковариантная производная от некоторого сечения.

В силу последнего определения образ оператора $\nabla_{\partial} / \partial t^{\alpha}$ состоит из потенциальных тензорных полей.

ТЕОРема 6. Пусть $\nabla$ - плоская связность на расслоении $\xi$. Тогда необходимым и достаточным условием потенииальности тензорного поля $y_{\alpha}^{i}$ является соотношение

$$
\nabla_{\partial / \partial t^{\beta}} y_{\alpha}^{i}=\nabla_{\partial / \partial t^{\alpha}} y_{\beta}^{i}
$$

Для доказательства перепишем (17) в виде пфафффовой системы уравнений

$$
d x=\left(W_{\alpha} x+y_{\alpha}\right) d t^{\alpha} .
$$

Используя (13), убеждаемся, что условие интегрируемости этой пфаффовой системы

$$
\frac{\partial}{\partial t^{\beta}}\left(W_{\alpha} x+y_{\alpha}\right)=\frac{\partial}{\partial t^{\alpha}}\left(W_{\beta} x+y_{\beta}\right)
$$

эквивалентны соотношению (18).

ЗАмЕчАнИЕ. Доказательство теоремы 6 можно получить также из формулы для тензора кривизны $R$ связности $\nabla$

$$
R(X, Y)=\nabla_{X} \nabla_{Y}-\nabla_{Y} \nabla_{X}-\nabla_{[X, Y]}
$$

Поскольку $R=0$ и поскольку коммутатор координатных векторных полей $\partial / \partial t^{\alpha}$ и $\partial / \partial t^{\beta}$ равен нулю, получаем, что операторы $\nabla_{\partial / \partial t^{\alpha}}$ и $\nabla_{\partial / \partial t^{\beta}}$ коммутируют, т.е. вторые ковариантные производные от любого сечения $x(\cdot)$ для плоской связности не зависят от порядка дифференцирования.

Вновь заметим, что в случае, когда многообразие $\mathfrak{N}$ неодносвязно, локальное сечение $x(\cdot)$, являющееся потенциалом тензорного поля $y_{\alpha}^{i}$, при продолжении на $\mathfrak{N}$ может дать многозначную функцию.

Приведем теперь примеры использования развитой в данной статье техники. 
ПРИмеР 1. Рассмотрим функционал

$$
\begin{aligned}
\iint_{\mathfrak{N}}\left[\left(\frac{\partial x^{1}}{\partial t^{1}}\right)^{2}+\left(\frac{\partial x^{1}}{\partial t^{2}}\right)^{2}\right. & +\left(\frac{\partial x^{2}}{\partial t^{1}}\right)^{2}+\left(\frac{\partial x^{2}}{\partial t^{2}}\right)^{2} \\
& \left.+\left(1+\frac{1}{r^{2}}\right)\left(\left(x^{1}\right)^{2}+\left(x^{2}\right)^{2}\right)+\frac{2}{r} x^{1} x^{2}\right] d t^{1} \wedge d t^{2}
\end{aligned}
$$

где область интегрирования $\mathfrak{N}$ есть кольцо $\rho_{1} \leqslant r \leqslant \rho_{2},(\varphi, r)$ - полярные координаты, расслоение $\xi$ тривиальное.

Положительная определенность этого функционала очевидна. Однако, на этом примере мы покажем, что многозначному решению уравнения Эйлера (4) может отвечать однозначное, глобально определенное решение уравнения (8). Система уравнений Эйлера (4) имеет вид

$$
\begin{aligned}
& \Delta x^{1}-\left(1+\frac{1}{r^{2}}\right) x^{1}-\left(\frac{1}{r}\right) x^{2}=0, \\
& \Delta x^{2}-\left(1+\frac{1}{r^{2}}\right) x^{2}-\left(\frac{1}{r}\right) x^{1}=0 .
\end{aligned}
$$

Ее матричное решение есть многозначная функция

$$
u=e^{\varphi}\left(\begin{array}{ll}
\operatorname{ch} r & \operatorname{sh} r \\
\operatorname{sh} r & \operatorname{ch} r
\end{array}\right)
$$

Уравнение (8) имеет вид

$$
\frac{\partial W_{1}}{\partial t^{1}}+\frac{\partial W_{2}}{\partial t^{2}}+W_{1}^{2}+W_{2}^{2}-\left(\begin{array}{cc}
1+1 / r^{2} & 1 / r \\
1 / r & 1+1 / r^{2}
\end{array}\right)=0
$$

Решение этого уравнения мы найдем, используя найденное решение уравнения Эйлера

$$
\begin{aligned}
& W_{1}=\frac{\partial U}{\partial t^{1}} U^{-1}=\left(\begin{array}{cc}
-t^{2} / r^{2} & t^{1} / r \\
t^{1} / r & -t^{2} / r^{2}
\end{array}\right) \\
& W_{2}=\frac{\partial U}{\partial t^{2}} U^{-1}=\left(\begin{array}{cc}
t^{1} / r^{2} & t^{2} / r \\
t^{2} / r & t^{1} / r^{2}
\end{array}\right) .
\end{aligned}
$$

Это решение однозначно. Нетрудно проверить, что связность, определяемая этим решением, плоская. Действительно, матрицы $W_{1}$ и $W_{2}$ коммутируют и $\frac{\partial W_{1}}{\partial t^{2}}-\frac{\partial W_{2}}{\partial t^{1}}=0$, следовательно, соотношение (13) вьполнено.

ПримеР 2. Пусть $\mathfrak{N}$ - односвязная область и $\xi=\mathfrak{N} \times \mathbb{R}^{1}$. В этом случае $W_{\alpha}-$ скалярные функции и условие интегрируемости (13) принимает вид

$$
\frac{\partial W_{\beta}}{\partial t^{\alpha}}=\frac{\partial W_{\alpha}}{\partial t^{\beta}} .
$$

Последнее означает, что дифференциальная форма $W_{\alpha} d t^{\alpha}$ замкнута и в односвязном случае точна, т.е. существует функция $S(t)$ такая, что $\frac{\partial S}{\partial t^{\alpha}}=W_{\alpha}$. Тогда система уравнений (8) сводится к одному квадратичному уравнению с эллиптической главной частью

$$
\frac{\partial}{\partial t^{\alpha}}\left(A^{\alpha \beta} \frac{\partial S}{\partial t^{\beta}}\right)+A^{\alpha \beta} \frac{\partial S}{\partial t^{\alpha}} \frac{\partial S}{\partial t^{\beta}}+B-\frac{\partial C^{\alpha}}{\partial t^{\alpha}}=0 .
$$


ПримеР 3 (конкретизация примера 1). Пусть в предыдущем примере $A^{\alpha \beta}=\delta^{\alpha \beta}$ есть единичная матрица; $C^{\alpha}=0 ; B=-\omega^{2}$. Тогда уравнение (19) принимает вид

$$
\sum_{\alpha}\left[\frac{\partial^{2} S}{\left(\partial t^{\alpha}\right)^{2}}+\left(\frac{\partial S}{\partial t^{\alpha}}\right)^{2}\right]+\omega^{2}=0
$$

Уравнение (20) допускает частные решения вида

$$
S=k+\ln \left|\sin \left(\omega t^{\gamma}+L\right)\right| .
$$

Следовательно, если область $\mathfrak{N}$ лежит между двумя параллельными плоскостями $\Pi_{1}, \Pi_{2}$, расстояние между которыми меньше $\pi / \omega$, то независимо от размеров и формы области уравнение (20) имеет решение, определенное на всей области $\mathfrak{N}$, что гарантирует положительную определенность функционала

$$
\int_{\mathfrak{N}}\left[\sum_{\alpha}\left(\frac{\partial x}{\partial t^{\alpha}}\right)^{2}-\omega^{2} x^{2}\right] d t
$$

Действительно, уравнение (20) инвариантно относительно ортогональных преобразований, что позволяет перевести плоскости $\Pi_{1}, \Pi_{2}$ в координатные плоскости и использовать решение (21), определяюшее поле экстремалей в $\mathfrak{N}$.

Чтобы сформулировать более общий результат, введем следующее определение. Пусть $\mathscr{L}_{q}$ - прямая $t=l s+q$ с направляющим вектором $l$, где $s \in \mathbb{R}^{1}-$ параметр на этой прямой; $q \in \mathbb{R}^{\nu}$. Обозначим через $d_{q l}(\mathfrak{N})$ длину минимального отрезка, содержашего $\mathscr{L}_{q} \cap \mathfrak{N}$.

ОПРЕДЕЛЕНИЕ. ШІириной области $\mathfrak{N}$ в направлении вектора $l$ назовем

$$
d_{l}(\mathfrak{N})=\sup _{q \in \mathbb{R}^{\nu}} d_{q l}(\mathfrak{N})
$$

УТВЕРЖ ДЕНИЕ 2. Если $d_{l}(\mathfrak{N}) \leqslant \frac{\pi}{\omega}-\varepsilon$, әде $0<\varepsilon<\frac{\pi}{\omega}$, то функционал неотрицательно определен.

ДокАЗАТЕльство. Запишем уравнение (8) для функционала (22)

$$
\sum_{\alpha} \frac{\partial}{\partial t^{\alpha}} W_{\alpha}=-\omega^{2}-\sum_{\alpha} W_{\alpha}^{2}
$$

Совершим в $\mathbb{R}^{\nu}$ ортогональное преобразование, переводяшее $l$ в ось $O t^{1}$. Уравнение (23) при этом преобразовании сохраняется. Левая и правая границы области в новых координатах могут быть записаны в виде $t^{1}=g^{-}\left(t^{2}, \ldots, t^{\nu}\right), t^{1}=g^{+}\left(t^{2}, \ldots, t^{\nu}\right)$, причем $0 \leqslant g^{+}-g^{-} \leqslant \frac{\pi}{\omega}-\varepsilon$. Функции $W_{1}=\omega \operatorname{ctg} \omega\left(t^{1}-g^{-}\left(t^{2}, \ldots, t^{\nu}\right)+\varepsilon\right), W_{\gamma}=0$ при $\gamma>1$ дают решение уравнения (23), определенное при всех $t \in \mathfrak{N}$. По теореме 2 функционал (22) неотрицателен.

Заметим, что найденное решение не удовлетворяет условию интегрируемости (13), и определяемая им связность имеет ненулевую кривизну. Это означает, что нельзя построить (даже многозначного) поля экстремалей, порожденного данной связностью. Тем не менее достаточное условие получается в терминах решения уравнения (8). 
ПРимеР 4. Рассмотрим функционал

$$
\int_{\mathfrak{N}}\left[\left(\frac{\partial x^{1}}{\partial t^{1}}\right)^{2}+\cdots+\left(\frac{\partial x^{1}}{\partial t^{\nu}}\right)^{2}+\cdots+\left(\frac{\partial x^{n}}{\partial t^{1}}\right)+\cdots+\left(\frac{\partial x^{n}}{\partial t^{\nu}}\right)^{2}+B_{i j} x^{i} x^{j}\right] d t^{1} \wedge \cdots \wedge d t^{\nu},
$$

где $B$ постоянная симметрическая матрица, спектр которой лежит правее точки $-\lambda,(\lambda>0) ; \xi=\mathbb{R}^{n} \times \mathfrak{N}$.

УТВеРЖДЕНИЕ 3 . Предположим, что $d_{l}(\mathfrak{N})<\frac{\pi}{\lambda}-\varepsilon$. Тогда функиионал (24) неотрицателен.

ДоказАТЕЛЬСТво. Уравнение (8) для функционала (24) имеет вид

$$
\left(\frac{\partial W_{1}}{\partial t^{1}}\right)^{2}+\cdots+\left(\frac{\partial W_{\nu}}{\partial t^{\nu}}\right)^{2}=B-W_{1}^{T} W_{1}-\ldots-W_{\nu}^{T} W_{\nu}
$$

Как и в предыдушем примере, переведем ортогональным преобразованием направление $l$ в ось $O t^{1}$. Совершим теперь ортогональное преобразование в слоях $\mathbb{R}^{n}$, диагонализируюшее матрицу $B$. Положим $W_{\gamma}=0$ при $\gamma>1$ и будем искать диагональные решения уравнения $(25): W_{1}=\operatorname{diag}\left(v_{1}, \ldots, v_{n}\right)$. Имеем

$$
\dot{v}_{i}=-\lambda_{i}-v_{i}^{2}
$$

где $\lambda_{i}$ - собственные значения матрицы $B$. Рассмотрим решение системы (26)

$$
\begin{cases}v_{i}=\sqrt{\lambda_{i}} \operatorname{ctg} \sqrt{\lambda_{i}}\left(t^{1}-g^{-}\left(t^{2}, \ldots, t^{\nu}\right)+\varepsilon\right) & \text { при } \lambda_{i}>0, \\ v_{j}=\left(t^{1}-g^{-}\left(t^{2}, \ldots, t^{\nu}\right)+\varepsilon\right)^{-1} & \text { при } \lambda_{j}=0, \\ v_{k}=\sqrt{-\lambda_{k}} \operatorname{cth} \sqrt{-\lambda_{k}}\left(t^{1}-g^{-}\left(t^{2}, \ldots, t^{\nu}\right)+\varepsilon\right) & \text { при } \lambda_{k}<0 .\end{cases}
$$

Легко видеть, что это решение определено при всех $t \in \mathfrak{N}$, что доказывает неотрицательную определенность функционала (24).

\section{Список литературы}

1. Clebsch A. Über die zweite variation vielfache Integralen. // J. Reine Angew. Math. 1859. V. 56. P. $122-148$.

2. Арутюнов А. В. К теории вырожденных квадратичных форм классического вариационного исчисления // Изв. РАН. Сер. матем. 1994. Т. 58. №6. С. 3-50.

3. Terpstra F. J. Die darstellung biquadratischer Formen als Summen von Quadraten mit Anwendungen auf die Variationsrechnung // Math. Ann. 1938. V. 116. P. 166-180.

4. Матюхин Д. В. О положительно определенньх биквадратичных формах, непредставимых в виде суммы квадратов билинейных форм // Вест. МГУ. Сер. 1, матем., мех. 1995. № 2. C. 29-33.

5. Зеликин М. И. Оптимальное управление и вариационное исчисление. М.: Изд-во МГУ, 1985 .

6. Кобаяси ШІ., Номидзу К. Основы дифференциальной геометрии. М.: Наука, 1981.

7. Милнор Дж., Сташеф Дж. Характеристические классы. М.: Мир, 1979.

8. Deligne P. Equation differentielles a points singuliers reguliers // Lecture Notes in Math. № $163,1970$.

9. Атья М. Геометрия и физика узлов. М.: Мир, 1995.

Московский государственнњй

Поступила в редакцию

университет им. М.В. Ломоносова

16.04 .1996 